\title{
Cross-Layer Design for the Physical, MAC, and Link Layer in Wireless Systems
}

\author{
Petar Popovski, 1,2 Mary Ann Ingram, ${ }^{3}$ Christian B. Peel, ${ }^{4}$ \\ Shinsuke Hara, ${ }^{5}$ and Stavros Toumpis ${ }^{6}$ \\ ${ }^{1}$ Department of Electronic Systems, Aalborg University, Niels Jernes Vej 12, 9220 Aalborg Øst, Denmark \\ ${ }^{2}$ Oticon A/S, Kongebakken 9, 2765 Smørum, Denmark \\ ${ }^{3}$ School of Electrical and Computer Engineering, Georgia Institute of Technology, 777 Atlantic Drive NW, \\ Atlanta, GA 30332-0250, USA \\ ${ }^{4}$ ArrayComm LLC, San Jose, CA 95131-1014, USA \\ ${ }^{5}$ Graduate School of Engineering, Osaka City University, Osaka-shi 558-8585, Japan \\ ${ }^{6}$ Department of Electrical and Computer Engineering, University of Cyprus, P.O. Box 20537, 1678 Nicosia, Cyprus
}

Correspondence should be addressed to Petar Popovski, petarp@es.aau.dk

Received 14 August 2008; Accepted 14 August 2008

Copyright (c) 2009 Petar Popovski et al. This is an open access article distributed under the Creative Commons Attribution License, which permits unrestricted use, distribution, and reproduction in any medium, provided the original work is properly cited.

During the past decade, the research community and wireless practitioners have provided overwhelming evidence that the strictly layered ISO/OSI architecture can lead to largely suboptimal operation of wireless networks. The reasons for such operation are rooted in the defining features of wireless communication: the variable link quality and the fact that the wireless medium should be shared by multiple users. In order to properly reflect these features in the protocol stack, the methodology of cross-layer design has been adopted. In short, the cross-layer methodology allows certain important information to influence decisions in a layer that is originally not defined to use that information (e.g., the SNR information at the link layer or the queue size at the baseband module).

The motivation for this special issue is the observation that a cross-layer approach is particularly important when designing protocols at the physical (PHY) and medium access control (MAC)/link layer. This is because the defining wireless features listed above have their strongest impact on these layers and their interaction. In recent years, the importance of the cross-layer design at the PHY/MAC/link layer has been reiterated through the emergence of many innovative techniques, such as opportunistic communication, rate adaptation, cross-layered scheduling, and so on. Judging from those developments, we can expect that crosslayer solutions at the lowest protocol layers will have a decisive impact in future wireless networks.
This special issue consists of eleven manuscripts that clearly advance the state-of-the art in the area of wireless cross-layer design. They were rigorously selected out of the 29 submitted manuscripts and the reviewers have put much effort into supporting the review process that, for most of the manuscripts, had three iterations. We believe that we have assembled an excellent collection of contributions, which covers many pertinent aspects of the PHY/MAC/link layer protocol design and reveals to the public innovative mechanisms and analysis tools. We have grouped the manuscripts into four subtopics: (1) reporting/prediction of fading channels, (2) random access protocols, (3) downlink resource allocation, and (4) distributed resource allocation.

Two papers are centered on the features of the fading channel. Recent advances on the prediction of fading channels have enabled the reliable prediction of the state of a channel for travel distances on the order of a few wavelengths. Ashraf et al., in their work titled "Channel MAC protocol for opportunistic communication in ad hoc wireless networks," use this observation to develop a MAC protocol under which users transmit depending on their predictions of the quality of the channel between them and their destination. The proposed protocol achieves excellent performance in terms of both throughput, and, more impressively, fairness. This is yet another excellent, and highly original, example of the benefits that can be reaped if 
information from the PHY layer is allowed to influence the operation of the MAC layer.

The second paper, "Exploiting transmit buffer information at the receiver in block-fading channels" by Dinesh Rajan considers an interesting problem: how to use the transmit buffer information at the receiver (TBIR) when the transmitter has a partial channel state information. The author develops a design framework for systems that utilize feedback and feed-forward information in block fading channels. TBIR is used at the receiver to efficiently quantize and report the state of the fading channel back to the transmitter. The results show that this innovative approach can lead to a reduction of the packet loss as well as power savings.

The next four papers deal with random access protocols. The paper by Romaszko and Blondia, entitled "Cross layer PHY-MAC protocol for wireless static and mobile ad hoc networks," introduces a novel protocol for use in ad hoc networks which performs significantly better, in terms of delay, throughput, and fairness, with respect to other currently proposed solutions. The improvement is achieved by the joint use of a power control algorithm on the PHY layer and a backoff algorithm on the MAC layer. The protocol is an excellent example of the gains to be had by a joint design across these two layers.

The paper "Further development of synchronous array method for ad hoc networks," by Yu et al., focuses on the scheduling and spacing of cochannel transmissions within a multihop ad hoc network with low mobility. It extends the evaluation of the opportunistic synchronous array method (O-SAM) protocol, which adapts to channel gain variations within a local area or subnet, to general loading conditions and multiple antennas per node. It also introduces a distributed synchronous array method (DSAM), which combines multislot contention in a control frame with a "cooperation radius." D-SAM offers insights into the fundamental throughput of the 802.16 MSH-DSCH protocol under low mobility.

In ad hoc networks, the collision avoidance medium access control (MAC) protocol allowing a single communication link at a time is not always beneficial, because it limits the utilization of the spatial resource. The paper by Kusume et al. entitled "Medium access in spread spectrum ad hoc networks with multiuser detection" proposes an integrated design of MAC and physical (PHY) protocols based on multiuser detection (MUD) to increase the throughput of ad hoc networks. The proposed MUD-MAC protocol outperforms the IEEE 802.11 carrier sense multiple access with collision avoidance (CSMA/CD) protocol in terms of the overall throughput.

Random packet CDMA (RP-CDMA) is a recent crosslayer technique that reduces the probability of collisioninduced packet errors by applying multiuser detection. In "Enhancing the performance of random access networks with random packet CDMA and joint detection," Kempter et al. provide a thorough analysis of the RP-CDMA, by taking into account realistic physical-layer limitations. The authors consider the two virtual channels created over the wireless channel: the header channel and the data channel, respectively. The system is evaluated for different multiuser receiver structures in term of throughput and queue sizes. This paper confirms that the generalization of the notion of collision through multiuser decoding is an exemplary topic of the cross-layer design between the physical and the medium access layer.

Four papers are dedicated to different scenarios/aspects of the downlink resource allocation. Finding the capacity of MIMO broadcast channels, a long standing problem in the information theory community, was recently solved. Brehmer and Utschick, in their work entitled "Nonconcave utility maximization in the MIMO broadcast channel," use this recently acquired knowledge to develop an algorithm for performing nonconcave utility maximization in such channels. They make use of the rich structure of the capacity region, to solve this tough problem in two steps: in the first step, the optimal point on the capacity region is determined, and on the second step the optimum parameter setup is found.

Zhou and Wunder introduce a scheduling policy which decomposes the cross-layer delay optimization problem into two subproblems: allocation of physical resources and user priority management. In their paper "Throughput-optimal scheduling with low average delay for cellular broadcast systems," the first subproblem is translated into a weighted sum rate maximization problem that can be efficiently solved for different channel models. For the second subproblem the authors present an algorithm which finds delay-minimizing rate weights. Simulations show that this algorithm gives low delay and maximum throughput.

For orthogonal frequency division multiple access (OFDMA) systems, resource allocation, namely, how to provide adequate subcarriers and data rates while satisfying each user's QoS requirement is a challenging problem. The optimal solution assuming full and perfect channel state information (CSI) on each user is known, but it requires not only more computational complexity but also more bandwidth and time delay on feedback channels. The paper by Alsawah and Fijalkow entitled "Practical radio link resource allocation for fair QoS-provision on OFDMA downlink with partial channel-state information" proposes a practical resource allocation method utilizing partial CSI of each user's average channel gain for an OFDMA single-cell down link. As compared with the optimal method with full and perfect CSI, the proposed method offers little spectral efficiency degradation with the much lower computational complexity and feedback overhead.

Downlink resource allocation for OFDMA-type systems with relays is treated in the paper "A coordinated resource allocation algorithm for infrastructure-based relay networks" by Müller et al. The authors propose an allocation algorithm based on the channel state information (CSI) at the transmitting base station (BS) and at the transmitting relay stations (RSs). The cross-layered approach plays a major role in reconciling the realistic amount of signaled CSI at the transmitters with the throughput demands set by the users. The extensive evaluations confirm that the proposed algorithm is superior to the existing approaches and succeeds in maximizing the sum rate in a cell, while providing each 
user with the minimal requested data rate and the requested tolerable error probability.

The last paper considers issues related to distributed resource allocation. In "Adaptive cross-layer distributed energy-efficient resource allocation algorithms for wireless data networks," Buzzi et al. address the problem of adaptive and distributed implementation of noncooperative games for energy-efficient resource allocation. Readily available measurements, such as the received data, are utilized for the adaptive and distributed resource allocation policies presented. Their stochastic non-cooperative algorithms for power allocation, spreading code allocation, and choice of the uplink (linear) receiver are shown via simulation to approach the performance of cooperative techniques which also assume perfect parameter knowledge.

\section{Acknowledgments}

The editors would like to thank all the reviewers for their efforts in making this special issue. They would also like to thank the Editor-in-Chief, and the editorial staff of Hindawi Publishing Corporation.

Petar Popovski Mary Ann Ingram Christian B. Peel Shinsuke Hara Stavros Toumpis 